\title{
Magnetic Monopoles in Multivector Boson Theories
}

\author{
Koichiro Kobayashi, ${ }^{1}$ Nahomi Kan, ${ }^{2}$ and Kiyoshi Shiraishi ${ }^{3}{ }^{3}$ \\ ${ }^{1}$ National Institute of Technology, Oshima College, Suooshima-cho, Yamaguchi 742-2193, Japan \\ ${ }^{2}$ National Institute of Technology, Gifu College, Motosu-shi, Gifu 501-0495, Japan \\ ${ }^{3}$ Graduate School of Sciences and Technology for Innovation, Yamaguchi University, Yamaguchi-shi, Yamaguchi 753-8512, Japan
}

Correspondence should be addressed to Kiyoshi Shiraishi; shiraish@yamaguchi-u.ac.jp

Received 1 February 2018; Revised 23 May 2018; Accepted 30 May 2018; Published 21 June 2018

Academic Editor: Luca Stanco

Copyright (C) 2018 Koichiro Kobayashi et al. This is an open access article distributed under the Creative Commons Attribution License, which permits unrestricted use, distribution, and reproduction in any medium, provided the original work is properly cited. The publication of this article was funded by SCOAP ${ }^{3}$.

A classical solution for a magnetic monopole is found in a specific multivector boson theory. We consider the model whose $[S U(2)]^{N+1}$ gauge group is broken by sigma model fields (à la dimensional deconstruction) and further spontaneously broken by an adjoint scalar (à la triplet Higgs mechanism). In this multivector boson theory, we find the solution for the monopole whose mass is $M_{N} \sim(4 \pi v / g) \sqrt{N+1}$, where $g$ is the common gauge coupling constant and $v$ is the vacuum expectation value of the triplet Higgs field, by using a variational method with the simplest set of test functions.

\section{Introduction}

The existence of magnetic monopoles (for reviews, see [1-6]) has been discussed for many years, although monopoles have not yet been observed experimentally.

In 1931, Dirac [7] reconsidered the duality in electromagnetism and showed that the quantum mechanics of an electrically charged particle can be consistently formulated in the presence of a point magnetic charge, provided that the magnetic charge $g_{m}$ is related to the electric charge $e$ by $e g_{m}=$ $n \hbar c / 2$ with an integer $n$. In 1974, 't Hooft [8] and Polyakov [9] found that a nonsingular configuration arises from spontaneous symmetry breaking in a certain class of non-Abelian gauge theory. Their models are based on the Georgi-Glashow model [10], which uses spontaneous symmetry breaking of $S U(2)$ gauge symmetry by a scalar field in the adjoint representation. The 't Hooft-Polyakov monopoles are classical solutions, which are stable for topological reasons. Recently, the mathematical study of monopoles has focused on not only topology, but also integrable systems, supersymmetry, nonperturbative analyses, and so on.

In the present paper, we consider a novel monopole in a multivector boson theory, which is based on dimensional deconstruction [11, 12] and the Higgsless theories [13-18]. The Higgsless theory is one of the theories that include symmetry breaking of the electroweak symmetry. In the Higgsless theory, for example, the $[S U(2)]^{N} \otimes U(1)$ gauge theory is considered. Such a theory yields $N$ sets of massive vector fields besides one massless photon field.

In our model of the multivector boson theory, $[S U(2)]^{N+1}$ gauge symmetry is assumed. One of the $S U(2)$ gauge groups is broken by an adjoint scalar as in the Georgi-Glashow model. There remains one massless vector field due to the triplet Higgs mechanism. We can thus construct the ' $t$ Hooft-Polyakov-type monopole configuration in the model. We estimate the monopole mass $M \sim(4 \pi v / g) \sqrt{N+1}$, where $v$ is the vacuum expectation value of the scalar field, and $g$ is the coupling constant of the gauge field.

In Section 2, we briefly review dimensional deconstruction and the Higgsless theory. Our model of the multivector boson theory is shown in Section 3, which is a generalization of the gauge-field part of the Higgsless theory. The mass spectrum in the multivector boson theories is investigated in Section 4. In Section 5, we demonstrate the construction of monopole configurations in the multivector boson theory. In order to treat many variables, we propose an approximation scheme by a variational method in this section. In Section 6, we discuss the magnetic charge of the monopole in the multivector boson theory. The final section (Section 7) is devoted to summary and discussion. 


\section{Deconstruction and Higgsless Theory}

We review the basic idea of dimensional deconstruction [11, 12] and the Higgsless theories [13-18] in this section. We consider $N+1$ gauge fields $A_{1 \mu}, A_{2 \mu}, \ldots A_{N+1, \mu}$. The field strength $G_{I \mu \nu}(I=1,2, \ldots, N+1)$ is defined as

$$
G_{I \mu \nu} \equiv \partial_{\mu} A_{I \nu}-\partial_{\nu} A_{I \mu}-i g_{I}\left[A_{I \mu}, A_{I \nu}\right]
$$

where $g_{I}$ is the $I$-th gauge coupling constant. The $I$-th field strength transforms as

$$
G_{I \mu \nu} \longrightarrow U_{I} G_{I \mu \nu} U_{I}^{\dagger} \quad(1 \leq I \leq N+1),
$$

according to the $I$-th gauge group transformation $U_{I} \in G_{I}$.

In addition to the gauge fields, we introduce $N$ scalar fields $\Sigma_{1}, \Sigma_{2}, \ldots \Sigma_{N}$, which would supply the NambuGoldstone fields as nonlinear-sigma model fields. The scalar field $\Sigma_{I}(I=1,2, \ldots, N)$ transforms as in the bifundamental representation,

$$
\Sigma_{I} \longrightarrow U_{I} \Sigma_{I} U_{I+1}^{\dagger} \quad(1 \leq I \leq N) .
$$

(Here, we show the case of "linear moose", and the different assignments of the transformation of $\Sigma_{I}$ yield the theory associated with various other types of moose diagrams [1118].)

Now, the Lagrangian density, which is invariant under the gauge transformation of $G_{1} \otimes G_{2} \otimes \cdots \otimes G_{N+1}$, is given by

$$
\mathscr{L}=-\frac{1}{2} \sum_{I=1}^{N+1} \operatorname{tr} G_{I \mu \nu} G_{I}^{\mu \nu}-\sum_{I=1}^{N} \operatorname{tr}\left(D_{\mu} \Sigma_{I}\right)^{\dagger}\left(D^{\mu} \Sigma_{I}\right),
$$

where the covariant derivative of $\Sigma_{I}$ is

$$
D_{\mu} \Sigma_{I} \equiv \partial_{\mu} \Sigma_{I}-i g_{I} A_{I \mu} \Sigma_{I}+i g_{I+1} \Sigma_{I} A_{I+1, \mu}
$$

and then its gauge transformation is

$$
D_{\mu} \Sigma_{I} \longrightarrow U_{I}\left(D_{\mu} \Sigma_{I}\right) U_{I+1}^{\dagger} .
$$

In the usual dimensional deconstruction scheme, we consider that $G_{1}=G_{2}=\cdots=G_{N+1}=G$ and $g_{1}=g_{2}=$ $\cdots=g_{N+1}=g$. We also assume that the absolute value of each nonlinear sigma model field $\left|\Sigma_{I}\right|$ has a common vacuum value, $f$. Then, the field $\Sigma_{I}$ is expressed as

$$
\Sigma_{I}=f \exp \left(i \frac{\pi^{a} T^{a}}{f}\right)
$$

where $T^{a}$ is the generator in the adjoint representation of $G$ and $\pi^{a}$ is the Nambu-Goldstone field, which is absorbed into the gauge fields. Taking the unitary gauge $\Sigma_{I}=f \times$ (identity matrix), we find that the kinetic terms of $\Sigma_{I}$ lead to the mass terms of the gauge fields as (provided that $\left.\operatorname{tr}\left(T^{a} T^{b}\right)=(1 / 2) \delta^{a b}\right)$

$$
\sum_{I=1}^{N} \operatorname{tr}\left(D_{\mu} \Sigma_{I}\right)^{\dagger}\left(D^{\mu} \Sigma_{I}\right)=\frac{1}{2} g^{2} f^{2} \sum_{I=1}^{N}\left(A_{I \mu}^{a}-A_{I+1, \mu}^{a}\right)^{2},
$$

and these produce the mass spectrum of vector bosons. It is known that a certain continuum limit of this model can be taken, which corresponds to the $G$ gauge theory with onedimensional compactification on to $S^{1} / Z_{2}$ (or an "interval").

In the Higgsless theories, for example, the gauge group $[S U(2)]^{N} \otimes U(1)$ is adopted for explaining the electroweak sector in the particle theory. Namely, we set $G_{1}=U(1)$ and $G_{2}=\cdots=G_{N+1}=S U(2)$. Then, the covariant derivative of $\Sigma_{1}$ is

$$
D_{\mu} \Sigma_{1} \equiv \partial_{\mu} \Sigma_{1}-i g_{1} A_{1 \mu} T^{3} \Sigma_{1}+i g \Sigma_{1} A_{2 \mu},
$$

where $g_{1}$ is the $U(1)$ gauge coupling constant, $g$ is the common $S U(2)$ gauge coupling constant, $A_{1 \mu}$ is the $U(1)$ gauge field, and $T^{3}$ is the third generator of $S U(2)$. The nonzero vacuum expectation value of $\Sigma_{I}$ leads to symmetry breaking $[S U(2)]^{N} \otimes U(1) \rightarrow U(1)[13-18]$, and we get only one massless electromagnetic field and $N$ sets of massive weak boson fields.

The original motivation for the Higgsless theory has been abandoned after the discovery of the Higgs particles. Nevertheless, we would like to extend the standard model, since there might be a lack of unknown extra particles, which explain the dark matter problem $[19,20]$. As a model of dark matter, the multivector boson theory describes a hidden sector of dark photons $[21,22]$ with mutual mixings. Therefore, we suppose that it is worth considering the theoretical models whose massive particle contents are rich and governed by certain symmetries.

\section{Multivector Boson Theory from the Higgsless Theory Incorporating the Higgs Mechanism}

Here, we consider the model whose $[S U(2)]^{N} \otimes U(1)$ gauge group comes from the spontaneous symmetry breaking by an adjoint scalar [10]: $[S U(2)]^{N+1} \rightarrow[S U(2)]^{N} \otimes U(1)$. The mechanism is now generally called the Higgs mechanism. The symmetry is broken into $U(1)$ by the vacuum expectation value of the nonlinear sigma model field $\Sigma_{I}$ introduced in the previous section. As a consequence, we have a monopole configuration; the construction of the monopole solution will be described in the next section. In this section, we define our model, and in the subsequent section, we show the mass spectrum of this model.

We consider the following Lagrangian density:

$$
\begin{aligned}
\mathscr{L}= & -\frac{1}{2} \sum_{I=1}^{N+1} \operatorname{tr} G_{I \mu \nu} G_{I}^{\mu \nu}-\sum_{I=1}^{N} \operatorname{tr}\left(D_{\mu} \Sigma_{I}\right)^{\dagger}\left(D^{\mu} \Sigma_{I}\right) \\
& -\operatorname{tr}\left(D_{\mu} \phi\right)^{\dagger}\left(D^{\mu} \phi\right)-\frac{\lambda}{4}\left(2 \operatorname{tr} \phi^{\dagger} \phi-v^{2}\right)^{2},
\end{aligned}
$$

where $G_{I \mu \nu}(I=1, \ldots, N+1)$ is the field strength of the $S U(2)_{I}$ gauge field $A_{I \mu}(I=1, \ldots, N+1)$ and $\Sigma_{I}(I=1, \ldots, N)$ is the nonlinear sigma model fields in the bifundamental representation of $S U(2)_{I} \otimes S U(2)_{I+1}$, which connect the gauge fields at neighboring sites, as in the dimensionally 
deconstructed model reviewed in the previous section. For simplicity, all the coupling constants of the gauge fields are assumed to be the same $g$.

Here, $\phi$ is a scalar field in the adjoint representation of $S U(2)_{1}$, and the covariant derivative of the scalar field $\phi$ is given by

$$
D_{\mu} \phi \equiv \partial_{\mu} \phi-i g\left[A_{1 \mu}, \phi\right]
$$

In the last term in the Lagrangian density (10), $\lambda$ is a positive constant and the constant $v$ is the scalar field vacuum expectation value.

First, we consider the symmetry breaking by the sigma fields. We choose the unitary gauge $\Sigma_{1}=\cdots=\Sigma_{N}=$ $f \times$ (the identity matrix). Then, the Lagrangian density is represented as follows:

$$
\begin{aligned}
\mathscr{L}= & -\frac{1}{4} \sum_{I=1}^{N+1} G_{I \mu \nu}^{a} G_{I}^{a \mu \nu}-\frac{1}{2} g^{2} f^{2} \sum_{I=1}^{N}\left(A_{I \mu}^{a}-A_{I+1, \mu}^{a}\right)^{2} \\
& -\frac{1}{2}\left(D_{\mu} \phi^{a}\right)\left(D^{\mu} \phi^{a}\right)-\frac{\lambda}{4}\left(\phi^{a} \phi^{a}-v^{2}\right)^{2}
\end{aligned}
$$

where

$$
\begin{aligned}
G_{I \mu \nu}^{a} & \equiv \partial_{\mu} A_{I \nu}^{a}-\partial_{\nu} A_{I \mu}^{a}+g \varepsilon^{a b c} A_{I \mu}^{b} A_{I \nu}^{c} \\
\text { and } D_{\mu} \phi^{a} & \equiv \partial_{\mu} \phi^{a}+g \varepsilon^{a b c} A_{1 \mu}^{b} \phi^{c} .
\end{aligned}
$$

Here, we use the component representations $A_{I \mu}=A_{I \mu}^{a} T^{a}$, $G_{I \mu \nu}=G_{I \mu \nu}^{a} T^{a}, \phi=\phi^{a} T^{a}$, and $D_{\mu} \phi=D_{\mu} \phi^{a} T^{a}$, and $\varepsilon^{a b c}$ is the totally antisymmetric symbol $(a=1,2,3)$.

Next, we consider the symmetry breakdown by the Higgs mechanism with respect to the adjoint scalar field $\phi$. We express the third component of the scalar field as $\phi^{3}=v+\varphi$. Then, the Lagrangian density is denoted by

$$
\begin{aligned}
\mathscr{L}= & -\frac{1}{4} \sum_{I=1}^{N+1} G_{I \mu \nu}^{1} G_{I}^{1 \mu \nu}-\frac{1}{2} g^{2} f^{2} \sum_{I=1}^{N}\left(A_{I \mu}^{1}-A_{I+1, \mu}^{1}\right)^{2} \\
& -\frac{1}{2} g^{2} v^{2} A_{1 \mu}^{1} A_{1}^{1 \mu}-\frac{1}{4} \sum_{I=1}^{N+1} G_{I \mu \nu}^{2} G_{I}^{2 \mu \nu} \\
& -\frac{1}{2} g^{2} f^{2} \sum_{I=1}^{N}\left(A_{I \mu}^{2}-A_{I+1, \mu}^{2}\right)^{2}-\frac{1}{2} g^{2} v^{2} A_{1 \mu}^{2} A_{1}^{2 \mu} \\
& -\frac{1}{4} \sum_{I=1}^{N+1} G_{I \mu \nu}^{3} G_{I}^{3 \mu \nu}-\frac{1}{2} g^{2} f^{2} \sum_{I=1}^{N}\left(A_{I \mu}^{3}-A_{I+1, \mu}^{3}\right)^{2} \\
& -\frac{1}{2} \partial_{\mu} \varphi \partial^{\mu} \varphi-\lambda \nu^{2} \varphi^{2}+(\text { interaction terms }),
\end{aligned}
$$

where the labels $a$ are explicitly represented. We have only one massless $U(1)$ symmetric gauge field in the third component. Therefore, we have obtained the symmetry breaking $S U(2) \rightarrow$ $U(1)$ by using the Higgs mechanism. This type of symmetry breaking gives rise to the 't Hooft-Polyakov monopole configuration.

It should be noted that we do not discuss which sequences of symmetry breaking, that is, $[S U(2)]^{N+1} \rightarrow[S U(2)]^{N} \otimes$ $U(1) \rightarrow U(1)$ or $[S U(2)]^{N+1} \rightarrow S U(2) \rightarrow U(1)$, occurred in the universe, although the order may have an effect on the process of creation of monopoles in the early universe.

\section{Mass Spectrum of Vector Bosons}

In the Lagrangian density (14), the mass term of gauge fields for $a=1$ is

$$
\begin{aligned}
\mathscr{L}_{\text {mass term } a=1} & =-\frac{1}{2} g^{2} f^{2} \sum_{I=1}^{N}\left(A_{I \mu}^{1}-A_{I+1, \mu}^{1}\right)^{2}-\frac{1}{2} g^{2} v^{2} A_{1 \mu}^{1} A_{1}^{1 \mu} \\
& =-\frac{1}{2} g^{2} f^{2}
\end{aligned}
$$$$
\times\left(\begin{array}{lllllll}
A_{1}^{1 \mu} & A_{2}^{1 \mu} & A_{3}^{1 \mu} & \cdots & A_{N-1}^{1 \mu} & A_{N}^{1 \mu} & A_{N+1}^{\mu}
\end{array}\right)\left(\begin{array}{cccccc}
1+\frac{v^{2}}{f^{2}} & -1 & & & \\
-1 & 2 & -1 & & \\
& -1 & 2 & -1 & \\
& & \ddots & \ddots & \ddots & \\
& & -1 & 2 & -1 & \\
& & & -1 & 2 & -1 \\
& & & -1 & 1
\end{array}\right)\left(\begin{array}{c}
A_{1 \mu}^{1} \\
A_{2 \mu}^{1} \\
A_{3 \mu}^{1} \\
\vdots \\
A_{N-1, \mu}^{1} \\
A_{N \mu}^{1} \\
A_{N+1, \mu}^{1}
\end{array}\right) .
$$ 
Therefore, for $a=1$, the mass-squared matrix $\left(\text { mass }^{1}\right)^{2}$ of the vector bosons is

$$
\begin{aligned}
& \left(\text { mass }^{1}\right)^{2} \\
& =g^{2} f^{2}\left(\begin{array}{ccccccc}
1+\frac{v^{2}}{f^{2}} & -1 & & & & \\
-1 & 2 & -1 & & & \\
& -1 & 2 & -1 & & \\
& & \ddots & \ddots & \ddots & \\
& & -1 & 2 & -1 & \\
& & & -1 & 2 & -1 \\
& & & & -1 & 1
\end{array}\right) \\
& \equiv g^{2} f^{2}\left(M^{1}\right)^{2} .
\end{aligned}
$$

We consider the eigenvalue equation

$$
\left(M^{1}\right)^{2} \mathbf{A}^{1}=\left(M_{E}^{1}\right)^{2} \mathbf{A}^{1}
$$

where $\mathbf{A}^{1}$ is the eigenvector

$$
\mathbf{A}^{1} \equiv\left(\begin{array}{c}
A_{1 \mu}^{1} \\
A_{2 \mu}^{1} \\
A_{3 \mu}^{1} \\
\vdots \\
A_{N-1, \mu}^{1} \\
A_{N \mu}^{1} \\
A_{N+1, \mu}^{1}
\end{array}\right),
$$

and $\left(M_{E}^{1}\right)^{2}$ is the eigenvalue.

We show the $M_{E}^{1}-v / f$ graphs in Figure 1. The highest eigenvalue behaves differently from the other eigenvalues. When $v / f \rightarrow \infty$, the highest eigenvalue becomes $M_{E}^{1} \sim v / f$, but the other eigenvalues asymptotically approach constant values that are less than two.

The mass term of gauge fields for $a=2$ is the same as for $a=1$, but the mass term is different for $a=3$. The masssquared matrix of gauge fields for $a=3$ is

$$
\begin{aligned}
\left(\text { mass }^{3}\right)^{2} & =g^{2} f^{2}\left(\begin{array}{ccccccc}
1 & -1 & & & & \\
-1 & 2 & -1 & & & \\
& -1 & 2 & -1 & & \\
& & \ddots & \ddots & \ddots & \\
& & -1 & 2 & -1 & \\
& & & -1 & 2 & -1 \\
& & & & -1 & 1
\end{array}\right) \\
& \equiv g^{2} f^{2}\left(M^{3}\right)^{2} .
\end{aligned}
$$

The eigenvalues $M_{E}^{3}$ can be analytically obtained [13] as

$$
\left(M_{E}^{3}\right)_{n}=2 \sin \frac{n \pi}{2(N+1)} \quad(n=0, \ldots, N) .
$$

Obviously, there is a zero mode, and we have only one massless vector field in the theory after symmetry breakdown.

\section{Energy and Equations of Motion of the Monopole in the Multivector Boson Theory}

In the multivector boson theory defined by the Lagrangian density (14), the 't Hooft-Polyakov-type monopole is expected.

Similar to the " $t$ Hooft-Polyakov monopole, the static and spherically symmetric monopole solution in the multivector boson theory is considered to be specified by the following ansatz:

$$
\begin{aligned}
\phi^{a} & =\delta_{i a} \frac{x^{i}}{g r^{2}} H(r), \\
A_{I 0}^{a} & =0, \\
A_{I i}^{a} & =\varepsilon_{a i j} \frac{x^{j}}{g r^{2}}\left[1-K_{I}(r)\right],
\end{aligned}
$$

and the boundary conditions on the function of the radial coordinate $r$ are

$$
\begin{aligned}
& \lim _{r \rightarrow 0} \frac{H(r)}{r}=0, \\
& \lim _{r \rightarrow \infty} \frac{H(r)}{r}=g v, \\
& \lim _{r \rightarrow 0} K_{I}(r)=1, \\
& \lim _{r \rightarrow \infty} K_{I}(r)=0 .
\end{aligned}
$$

The common form of $A_{I i}^{a}$ is due to the requirement of finite energy of the monopole, i.e., the contribution of the term (8) to the energy density vanishes at spatial infinity.

For the static case, the energy density is given by $-\mathscr{L}$. Substituting the ansatz, we obtain the expression for total energy

$$
\begin{aligned}
E_{N} & =\frac{4 \pi v}{g} \int_{0}^{\infty} d \xi\left[\sum_{I=1}^{N+1}\left\{\left(K_{I}^{\prime}\right)^{2}+\frac{1}{2 \xi^{2}}\left(1-K_{I}^{2}\right)^{2}\right\}\right. \\
& +\frac{f^{2}}{v^{2}} \sum_{I=1}^{N}\left(K_{I}-K_{I+1}\right)^{2}+\frac{1}{2}\left(H^{\prime}-\frac{H}{\xi}\right)^{2}+\frac{1}{\xi^{2}} K_{1}^{2} H^{2} \\
& \left.+\frac{\lambda \xi^{2}}{4 g^{2}}\left(\frac{H^{2}}{\xi^{2}}-1\right)^{2}\right]
\end{aligned}
$$

where we set $\xi \equiv g v r$ and the prime $\left(^{\prime}\right)$ denotes the derivative with respect to $\xi$. 

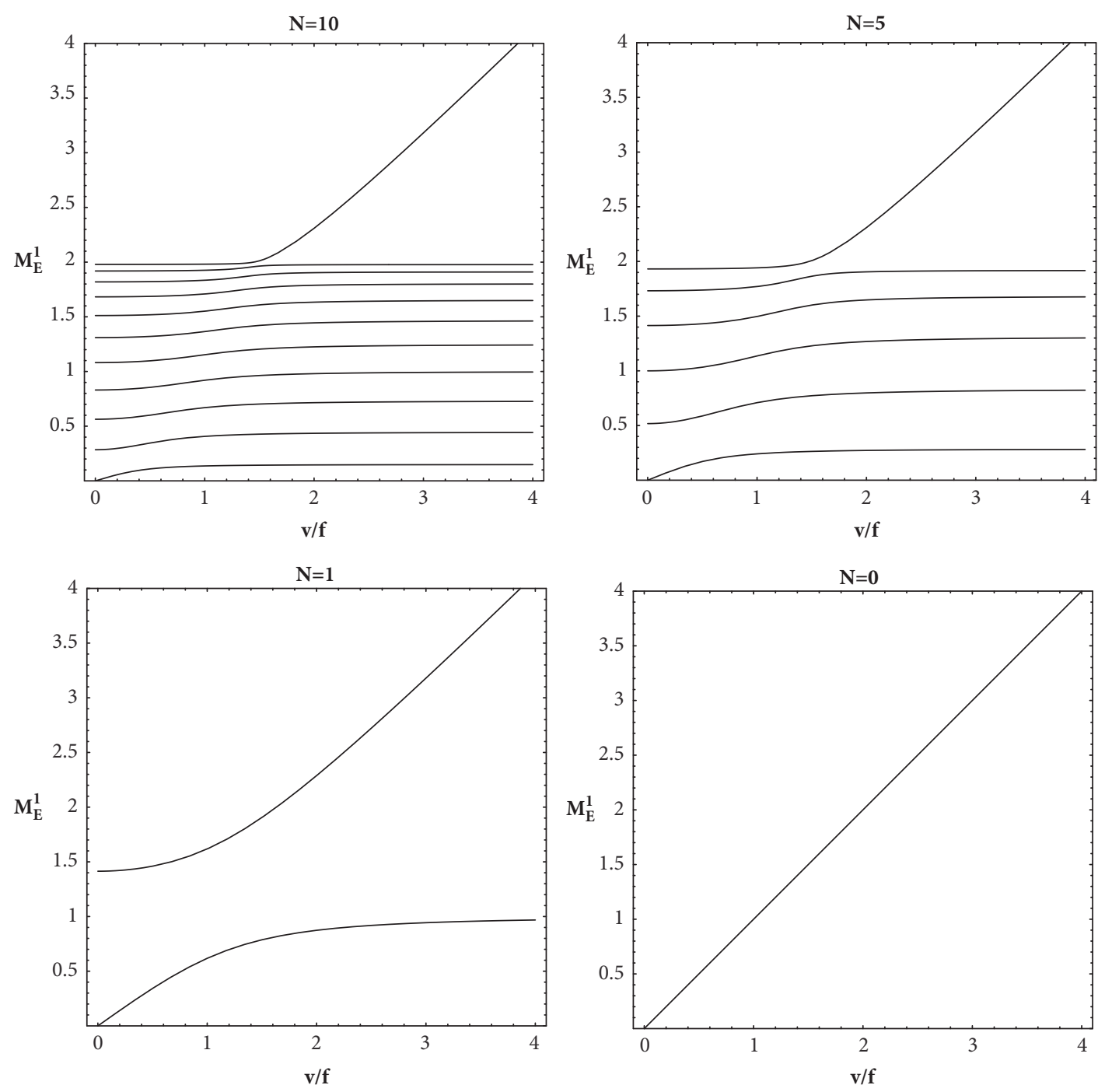

Figure 1: The eigenvalues $M_{E}^{1}$ are shown as functions of $v / f$ in the cases of $N=10,5,1$, and 0 .

From this expression, we can obtain the following equations of motion by the variational principle:

$$
\begin{gathered}
\xi^{2} K_{1}^{\prime \prime}=K_{1}\left(K_{1}^{2}-1\right)+H^{2} K_{1}+\frac{f^{2}}{v^{2}} \xi^{2}\left(K_{1}-K_{2}\right), \\
\xi^{2} K_{I}^{\prime \prime}=K_{I}\left(K_{I}^{2}-1\right)+\frac{f^{2}}{v^{2}} \xi^{2}\left(2 K_{I}-K_{I-1}-K_{I+1}\right) \\
\quad(2 \leq I \leq N), \\
\xi^{2} K_{N+1}^{\prime \prime}=K_{N+1}\left(K_{N+1}^{2}-1\right)+\frac{f^{2}}{v^{2}} \xi^{2}\left(K_{N+1}-K_{N}\right), \\
\xi^{2} H^{\prime \prime}=2 H K_{1}+\frac{\lambda}{g^{2}} H\left(H^{2}-\xi^{2}\right) .
\end{gathered}
$$

Analytical and semianalytical studies of the single ' $t$ Hooft-Polyakov monopole are found in [23-25]. Because it is hard to find a set of solutions for these coupled equations for large $N$ and because we are presently considering a simple toy model, we adopt a simple variational method to obtain approximate solutions in this paper. We have confirmed that this approach obtains a good solution for the 't Hooft-Polyakov monopole in the BPS limit.

For the approximation, we assume that the solutions take the following forms:

$$
\begin{aligned}
& K_{I}(\xi)=\left(1+a_{I} \xi\right) \exp \left(-a_{I} \xi\right) \quad(1 \leq I \leq N+1), \\
& \frac{H(\xi)}{\xi}=1-\exp (-\alpha \xi)
\end{aligned}
$$

where both $a_{I}(1 \leq I \leq N+1)$ and $\alpha$ are variational parameters. The functions $K_{I}(\xi)$ and $H(\xi)$ with minimal number of parameters apparently satisfy the boundary conditions and are similar to those of the solutions in the ' $t$ Hooft-Polyakov monopole. This is the reason why we assume the simple form of solutions as shown above. 

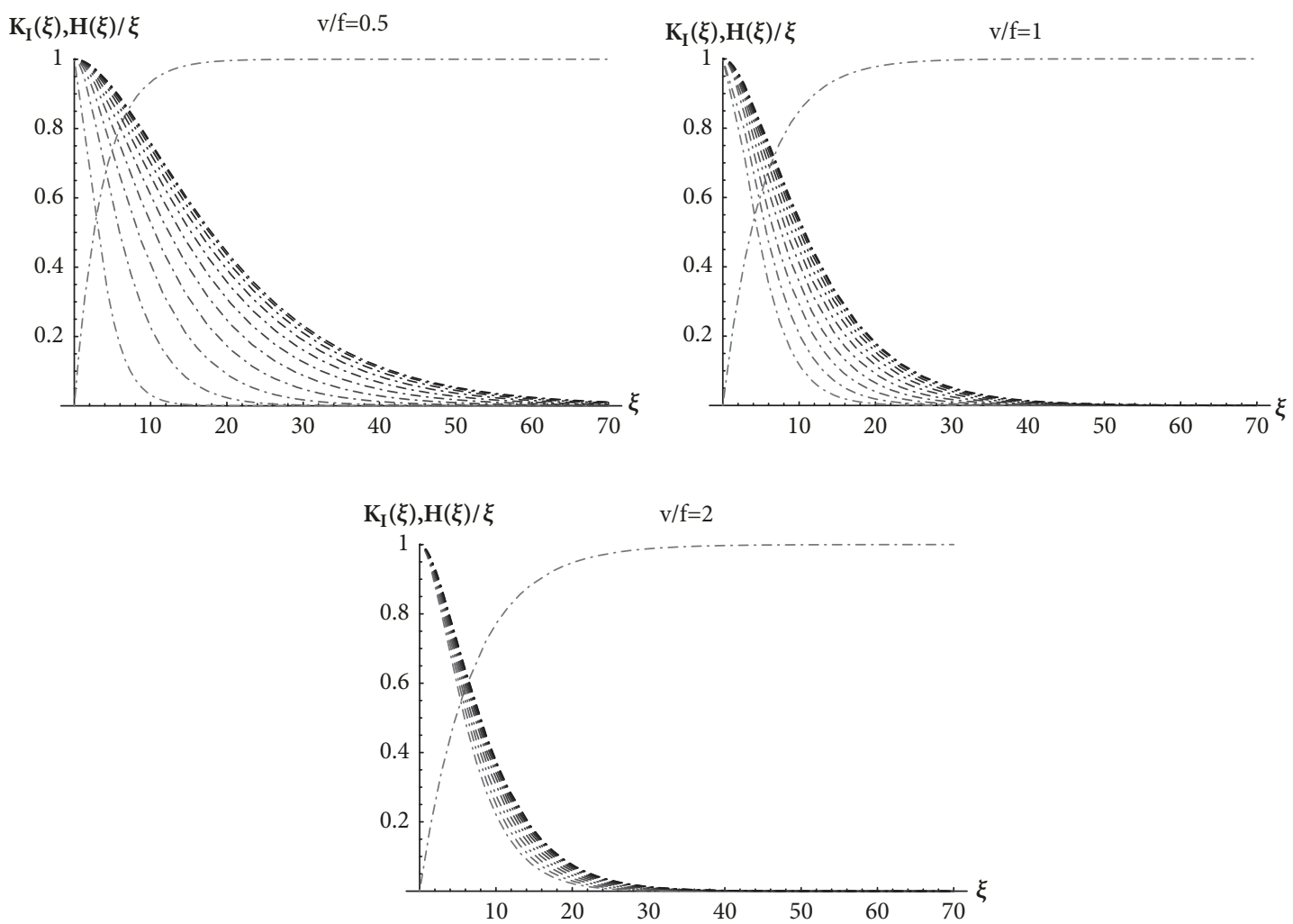

Figure 2: $K_{I}$ and $H / \xi$ are shown for $N=10, \lambda=0$. In each graph, $K_{I}<K_{I+1}(1 \leq I \leq N=10)$ at any $\xi$. The three graphs correspond to the cases of $f / v=0.5, f / v=1$, and $f / v=2$, respectively.

We substitute the expressions (30) and (31) into the energy $E_{N}$ and calculate the minimum value of the energy $E_{N}$ by varying the parameters $a_{I}$ and $\alpha$.

Each term is separately integrated as follows:

$$
\begin{aligned}
& \int_{0}^{\infty} d \xi\left\{\left(K_{I}^{\prime}\right)^{2}+\frac{1}{2 \xi^{2}}\left(1-K_{I}^{2}\right)^{2}\right\}=\frac{41}{64} a_{I}, \\
& \int_{0}^{\infty} d \xi\left(K_{I}-K_{I+1}\right)^{2} \\
& =\frac{5}{4 a_{I}}+\frac{5}{4 a_{I+1}}-\frac{4\left(a_{I}^{2}+3 a_{I} a_{I+1}+a_{I+1}^{2}\right)}{\left(a_{I}+a_{I+1}\right)^{3}}, \\
& \int_{0}^{\infty} d \xi\left(H^{\prime}-\frac{H}{\xi}\right)^{2}=\frac{1}{4 \alpha}, \\
& \int_{0}^{\infty} d \xi \frac{1}{\xi^{2}} K_{1}^{2} H^{2} \\
& =\frac{\alpha^{2}\left(56 a_{1}^{4}+132 a_{1}^{3} \alpha+111 a_{1}^{2} \alpha^{2}+39 a_{1} \alpha^{3}+5 \alpha^{4}\right)}{4 a_{1}\left(a_{1}+\alpha\right)^{3}\left(2 a_{1}+\alpha\right)^{3}}, \\
& \int_{0}^{\infty} d \xi \xi^{2}\left(\frac{H^{2}}{\xi^{2}}-1\right)^{2}=\frac{635}{864 \alpha^{3}} .
\end{aligned}
$$

Therefore, the energy expressed by the variational parameters becomes

$$
\begin{aligned}
E_{N} & =\frac{4 \pi v}{g}\left[\frac{41}{64} \sum_{I=1}^{N+1} a_{I}+\frac{f^{2}}{v^{2}}\right. \\
& \cdot \sum_{I=1}^{N}\left\{\frac{5}{4 a_{I}}+\frac{5}{4 a_{I+1}}-\frac{4\left(a_{I}^{2}+3 a_{I} a_{I+1}+a_{I+1}^{2}\right)}{\left(a_{I}+a_{I+1}\right)^{3}}\right\} \\
& +\frac{1}{8 \alpha} \\
& +\frac{\alpha^{2}\left(56 a_{1}^{4}+132 a_{1}^{3} \alpha+111 a_{1}^{2} \alpha^{2}+39 a_{1} \alpha^{3}+5 \alpha^{4}\right)}{4 a_{1}\left(a_{1}+\alpha\right)^{3}\left(2 a_{1}+\alpha\right)^{3}} \\
& \left.+\frac{635 \lambda}{3456 g^{2} \alpha^{3}}\right] .
\end{aligned}
$$

We evaluate the minimum value of this energy by numerical calculation with Mathematica [26]. Thus, we get the approximate solution of $K_{I}(\xi)$ and $H(\xi) / \xi$, and the case of $N=10$ and $\lambda=0$ is shown in Figure 2.

The region of nonvanishing $K_{I}$ can be interpreted as the region where the $I$-th massive vector bosons $(a=1,2)$ condensate. For larger values of $f / v$, the ranges of finite $K_{I}$ become narrower and degenerate, while the distance where $H / \xi \sim 1$ becomes larger. 
We obtain the energy of the monopole in the limiting case $\lambda / g^{2}=0$ for the cases where $N=0,1,5$, and 10 and $f / v=$ $0.5,1$, and 2 .

$$
\begin{array}{ll}
E_{0}=\frac{4 \pi v}{g} \times 1.05 \cdots, & (\text { BPS monopole }) \\
E_{1}=\frac{4 \pi v}{g} \times 1.41 \cdots, & \left(\frac{f}{v}=0.5\right) \\
E_{1}=\frac{4 \pi v}{g} \times 1.47 \cdots, & \left(\frac{f}{v}=1\right) \\
E_{1}=\frac{4 \pi v}{g} \times 1.48 \cdots, & \left(\frac{f}{v}=2\right) \\
E_{5}=\frac{4 \pi v}{g} \times 2.07 \cdots, & \left(\frac{f}{v}=0.5\right) \\
E_{5}=\frac{4 \pi v}{g} \times 2.37 \cdots, & \left(\frac{f}{v}=1\right) \\
E_{5}=\frac{4 \pi v}{g} \times 2.51 \cdots, & \left(\frac{f}{v}=2\right) \\
E_{10}=\frac{4 \pi v}{g} \times 2.47 \cdots, & \left(\frac{f}{v}=0.5\right) \\
E_{10}=\frac{4 \pi v}{g} \times 2.99 \cdots, & \left(\frac{f}{v}=1\right) \\
E_{10}=\frac{4 \pi v}{g} \times 3.32 \cdots, & \left(\frac{f}{v}=2\right)
\end{array}
$$

From these results, we roughly estimate that the energy of the monopole $(\lambda=0)$ is

$$
E_{N} \sim \frac{4 \pi v}{g} \sqrt{N+1} \quad(\lambda=0),
$$

since the difference that appears due to different $f / v$ is smaller than that due to different $N$. We find that our approximate values of the static energies for $\lambda=0$ are well fitted to $E_{N} \approx(4 \pi v / g) \times 1.94 \times W(0.62 N+0.96)$, where $W(x)$ is the Lambert $W$-function, which is slightly smaller than $(4 \pi v / g) \sqrt{1+N}$ for large $N$. This is in contrast to the rather large dependence of the profiles of solutions for $K_{I}$ and $H / \xi$ on $f / v$ (Figure 2).

On the other hand, we know the exact value of the energy of the BPS limit $[27,28]$ for the ' $t$ Hooft-Polyakov monopole, which corresponds to $E_{0}$ for $\lambda=0$, as

$$
E(\lambda=0)=\frac{4 \pi v}{g} .
$$

Comparing these values, we find that the energy of the BPS monopole in the multivector boson theory is obtained by replacing $g \rightarrow g / \sqrt{N+1}$ in that of the usual BPS monopole.
Note that we only show the case of $\lambda \rightarrow 0$. However, we confirmed that the energy of the monopole changes at most factor two for a finite value of $\lambda / g^{2}$ in general.

\section{Magnetic Charge of the Monopole}

In this section, we specify the magnetic charge of the monopole in the multivector boson theory obtained in the previous section. First of all, we should discuss the definition of electric charge. As in Section 3, if we choose $\phi^{3}=v$, the massless gauge field satisfies

$$
A_{1 \mu}^{3}=A_{1 \mu}^{3}=\cdots=A_{N+1, \mu}^{3} \equiv \frac{1}{\sqrt{N+1}} A_{\mu}^{3} .
$$

The normalization factor is determined by the canonical form of the Lagrangian density of this zero-mode field. Therefore, if the charged matter field is virtually coupled only to $A_{1 \mu}$, similar to that in the triplet Higgs field, the electric charge of the matter field $e$ becomes

$$
e=\frac{g}{\sqrt{N+1}}
$$

and the field strength satisfies $G_{1 \mu \nu}^{3}=G_{1 \mu \nu}^{3}=\cdots=G_{N+1, \mu \nu}^{3} \equiv$ $(1 / \sqrt{N+1}) G_{\mu \nu}^{3}$.

Now, we consider the magnetic field far from the monopole. The projection of the vacuum expectation values of the field strength $[1,3,6]$ is

$$
\begin{aligned}
\lim _{r \rightarrow \infty} F_{i j} & =\lim _{r \rightarrow \infty} \hat{\phi}^{a} G_{i j}^{a}=\lim _{r \rightarrow \infty} \frac{1}{\sqrt{N+1}} \sum_{I=1}^{N+1} \widehat{\phi}^{a} G_{I i j}^{a} \\
& =\frac{\sqrt{N+1}}{g}\left(-\varepsilon_{a i j} \frac{x^{a}}{r^{3}}\right),
\end{aligned}
$$

where $\widehat{\phi}^{a}=\phi^{a} / v$. Then, the magnetic field $B^{i}$ is asymptotically

$$
B^{i}=-\frac{\sqrt{N+1}}{g} \frac{x^{i}}{r^{3}}=-\frac{x^{i}}{e r^{3}} .
$$

Comparing this magnetic field representation with the magnetic field created by a point magnetic charge $g_{m}$

$$
B^{i}=\frac{g_{m}}{4 \pi} \frac{x^{i}}{r^{3}}
$$

the magnetic charge $g_{m}$ of our monopole is

$$
g_{m}=-\frac{4 \pi}{g} \sqrt{N+1}=-\frac{4 \pi}{e} .
$$

This relation is the same as that for the 't Hooft-Polyakov monopole.

The static energy of the monopole in the multivector boson theory that was described in the previous section can be rewritten as

$$
E_{N} \sim \frac{4 \pi v}{g} \sqrt{N+1}=\frac{4 \pi v}{e}
$$


which is the same as the mass of the 't Hooft-Polyakov monopole (or, the case of $N=0$ ).

\section{Summary and Discussion}

In this paper, we studied the static, spherically symmetric monopole solutions in the multivector boson theory with $N+1$ sets of vector bosons with the gauge coupling $g$. The theory includes two mass scales $f$ and $v$. We found that $3 N+2$ massive vector bosons and a single massless vector boson (of the electromagnetic field) appear according to the theory described in Section 4. We used a simple variational method to obtain approximate solutions in Section 5. The solution of $K_{I}$ shows that the regions of existence of massive vector fields have a multilayer structure, where massive bosons "stratify". Although the profile of condensation of the massive degrees of freedom is sensitive with respect to both $N$ and $f / v$, the mass of the monopole is approximately $E_{N} \sim$ $(4 \pi v / g) \sqrt{N+1}=4 \pi v / e$, where $e$ is the electric charge defined in the theory. It is necessary to conduct a more accurate investigation for obtaining the precise dependence of mass of the monopole on $N$.

The model used in this study is the simplest one; therefore, we would like to investigate more general models, which have different coupling constants for different gauge fields or have complicated mass matrices as in the clockwork theory [2934].

Another possible connection to a phenomenological model can be considered in a model with symmetry breakdown by a Higgs doublet, as in the standard model. In 1997, Cho and Maison [35] found an electroweak monopole solution in the standard model. The Cho-Maison monopole and its generalization have been studied further [36,37], and an experimental search for them is going on $[38,39]$. We wish to investigate the multivector boson theory with a doublet Higgs field and compare the properties of its monopoles with those of the Cho-Maison monopoles.

We also wish to study a scenario in which the monopoles in the multivector boson theory represent the dark matter in the universe. Since the present model of multivector boson theory has two symmetry breaking scales $f$ and $v$ and there can be various mass spectra of massive vector bosons as seen in Section 4, we need to perform a detailed study on the process of symmetry breaking and (time-dependent) monopole production.

\section{Data Availability}

No data were used to support this study.

\section{Conflicts of Interest}

The authors declare that there are no conflicts of interest regarding the publication of this paper.

\section{Acknowledgments}

The authors thank Hideto Manjo for useful comments on numerical estimations.

\section{References}

[1] P. Goddard and D. I. Olive, "Magnetic monopoles in gauge field theories," Reports on Progress in Physics, vol. 4, no. 9, p. 1357, 1978.

[2] R. Rajaraman, Solitons and Instantons, North-Holland, New York, NY, USA, 1987.

[3] V. Rubakov, Classical Theory of Gauge Fields, Princeton Univ. Press, Princeton, NJ, USA, 2002.

[4] N. Manton and P. Sutcliffe, Topological Solitons, Cambridge University Press, Cambridge, UK, 2004.

[5] Y. M. Shnir, Magnetic Monopoles, Springer, Berlin, Germany, 2005.

[6] E. J. Weinberg, Classical Solutions in Quantum Field Theory, Cambridge Univ. Press, Cambridge, UK, 2012.

[7] P. A. M. Dirac, "Quantised singularities in the electromagnetic field," Proceedings of the Royal Society, vol. 133, p. 60, 1931.

[8] G. 't Hooft, "Magnetic monopoles in unified gauge theories," Nuclear Physics B, vol. 79, pp. 276-284, 1974.

[9] A. M. Polyakov, "Particle spectrum in quantum field theory," JETP Letters, vol. 20, no. 6, pp. 194-195, 1974.

[10] H. Georgi and S. L. Glashow, "Unity of all elementary-particle forces," Physical Review Letters, vol. 32, no. 8, pp. 438-441, 1974.

[11] N. Arkani-Hamed, A. G. Cohen, and H. Georgi, "(De)constructing dimensions," Physical Review Letters, vol. 86, no. 21, pp. 4757-4761, 2001.

[12] C. T. Hill, S. Pokorski, and J. Wang, "Gauge invariant effective Lagrangian for Kaluza-Klein modes," Physical Review D: Particles, Fields, Gravitation and Cosmology, vol. 64, no. 10, 2001.

[13] H.-C. Cheng, C. T. Hill, S. Pokorski, and J. Wang, "Standard model in the latticized bulk," Physical Review D, vol. 2001, Article ID 065007, 64.

[14] C. Csáki, C. Grojean, H. Murayama, L. Pilo, and J. Terning, "Gauge theories on an interval: unitarity without a Higgs boson," Physical Review D: Particles, Fields, Gravitation and Cosmology, vol. 69, no. 5, Article ID 055006, 19 pages, 2004.

[15] C. Csáki, C. Grojean, L. Pilo, and J. Terning, "Towards a Realistic Model of Higgsless Electroweak Symmetry Breaking," Physical Review Letters, vol. 92, no. 10, 2004.

[16] C. Csaki, J. Hubisz, and P. Maede, TASI Lectures on Electroweak Symmetry Breaking from Extra Dimensions, World Scientific, Singapore, 2006.

[17] R. Foadi, S. Gopalakrishna, and C. Schmidt, "Higgsless electroweak symmetry breaking from theory space," Journal of High Energy Physics, vol. 0403, p. 042, 2004.

[18] H. Georgi, "Fun with Higgsless theories," Physical Review, vol. D71, p. 015016, 2005.

[19] G. Bertone, D. Hooper, and J. Silk, "Particle dark matter: evidence, candidates and constraints," Physics Reports, vol. 405, no. 5-6, pp. 279-390, 2005.

[20] S. Profume, An Introduction to Particle Dark Matter, World Scientific, Singapore, 2017.

[21] L. B. Okun, "Limits of electrodynamics: paraphotons?" Journal of Experimental and Theoretical Physics, vol. 56, p. 502, 1982.

[22] B. Holdom, "Two U(1)'s and epsilon charge shifts," Physics Letters B, vol. 166, pp. 196-198, 1986.

[23] T. W. Kirkman and C. K. Zachos, "Asymptotic analysis of the monopole structure," Physical Review D: Particles, Fields, Gravitation and Cosmology, vol. 24, no. 4, pp. 999-1004, 1981. 
[24] C. L. Gardner, "The 't Hooft-Polyakov monopole near the Prasad-Sommerfield limit," Annals of Physics, vol. 146, no. 1, pp. 129-148, 1983.

[25] P. Forgács, N. Obadia, and S. Reuillon, “ Publisher's Note: Numerical and asymptotic analysis of the 't Hooft-Polyakov magnetic monopole [Phys. Rev. D," Physical Review D: Particles, Fields, Gravitation and Cosmology, vol. 71, no. 11, 2005.

[26] Wolfram, http://www.wolfram.com/.

[27] E. Bogomol'nyi, “The stability of classical solutions," Soviet Journal of Nuclear Physics, vol. 24, pp. 449-454, 1976.

[28] M. Prasad and C. Sommerfield, "Exact classical solution for the 't Hooft Monopole and the Julia-Zee Dyon," Physical Review Letters, vol. 35, no. 12, p. 760, 1975.

[29] K. Choi and S. H. Im, "Realizing the relaxion from multiple axions and its UV completion with high scale supersymmetry," Journal of High Energy Physics, vol. 1601, p. 149, 2016.

[30] D. E. Kaplan and R. Rattazzi, "Large field excursions and approximate discrete symmetries from a clockwork axion," Physical Review D, vol. 93, Article ID 085007, 2016.

[31] G. F. Giudice and M. McCullough, "A clockwork theory," Journal of High Energy Physics, vol. 2017, p. 036, 2017.

[32] M. Farina, D. Pappadopulo, F. Rompineve, and A. Tesi, "The photo-philic QCD axion," Journal of High Energy Physics, vol. 2017, no. 1, 2017.

[33] A. Kehagias and A. Riotto, "Clockwork inflation," Physics Letters B, vol. 767, pp. 73-80, 2017.

[34] T. Hambye, D. Teresi, and M. H. Tytgat, "A clockwork WIMP," Journal of High Energy Physics, vol. 2017, no. 7, 2017.

[35] Y. Cho and D. Maison, "Monopole configuration in WeinbergSalam model," Physics Letters B, vol. 391, no. 3-4, pp. 360-365, 1997.

[36] K. Kim, J. H. Yoon, and Y. M. Cho, "Finite energy electroweak dyon," The European Physical Journal C, vol. 75, p. 67, 2015.

[37] Y. M. Cho, K. Kim, and J. H. Yoon, "Gravitationally coupled electroweak monopole," Physics Letters B, vol. 761, pp. 203-206, 2016.

[38] J. Ellis, N. E. Mavromatos, and T. You, "The price of an electroweak monopole," Physics Letters B, vol. 756, pp. 29-35, 2016.

[39] B. Acharya, J. Alexandre, S. Baines et al., "Search for Magnetic Monopoles with the MoEDAL Forward Trapping Detector in $13 \mathrm{TeV}$ Proton-Proton Collisions at the LHC," Physical Review Letters, vol. 118, Article ID 061801, 2017. 

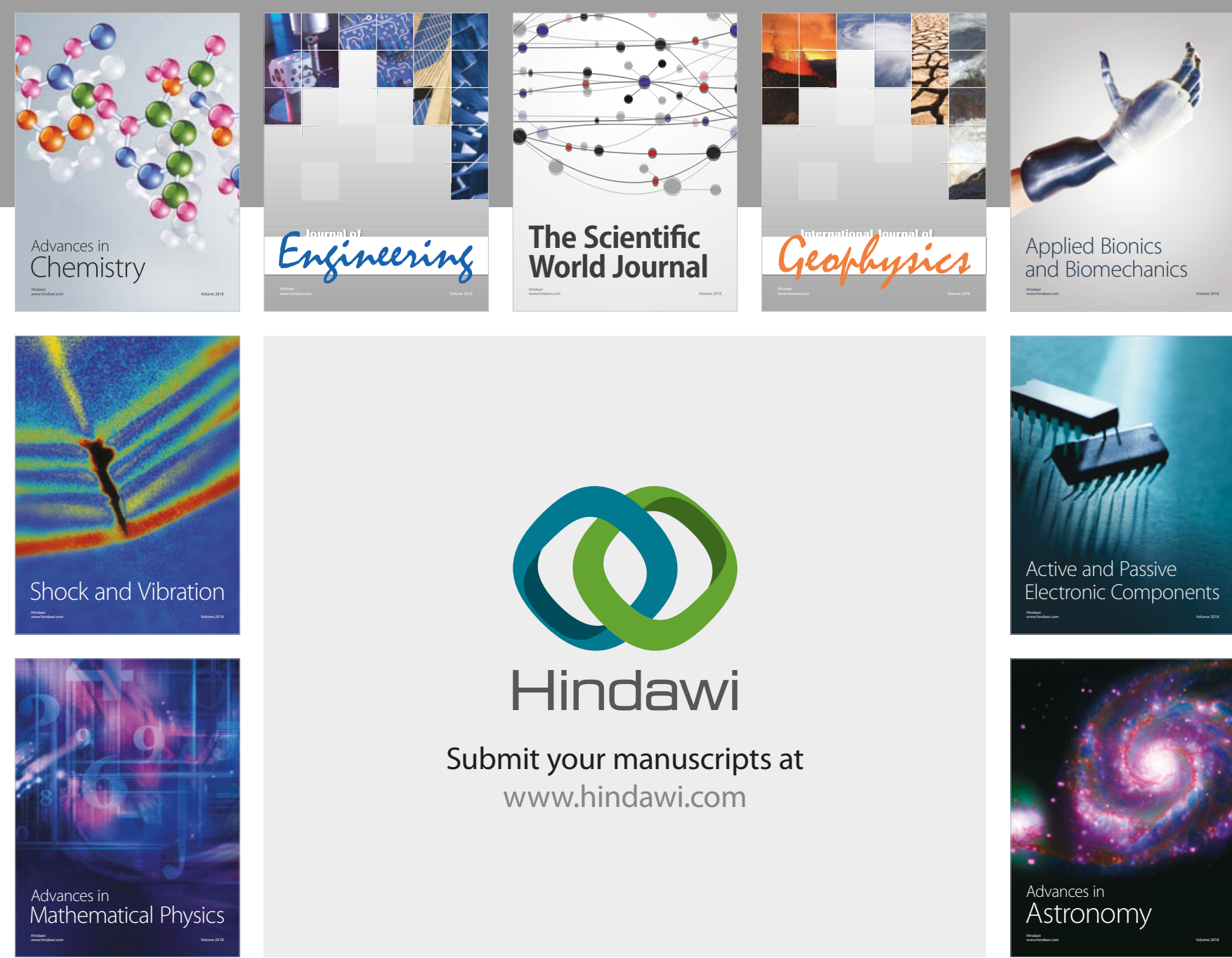

Submit your manuscripts at

www.hindawi.com

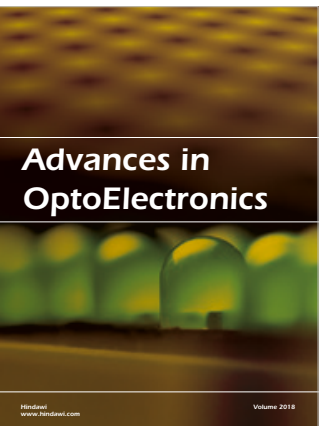

\section{Rotcting Machinery}
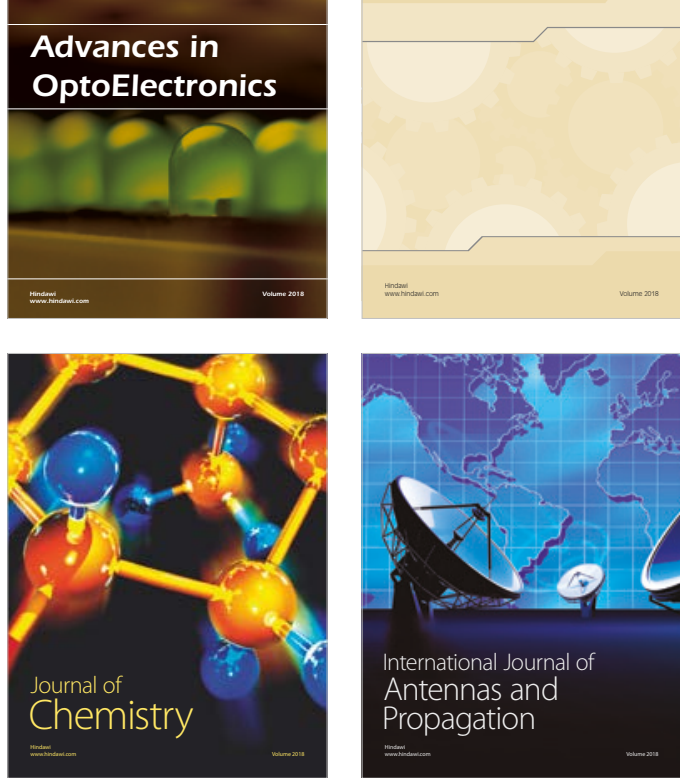

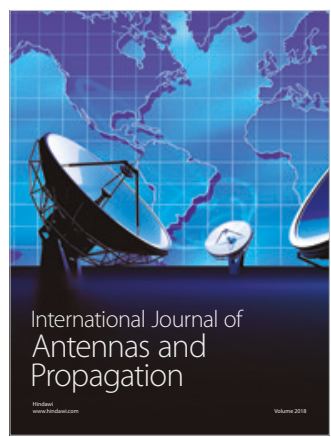

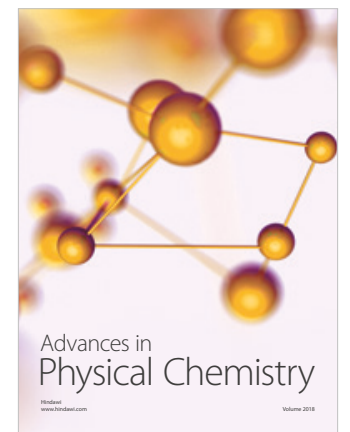

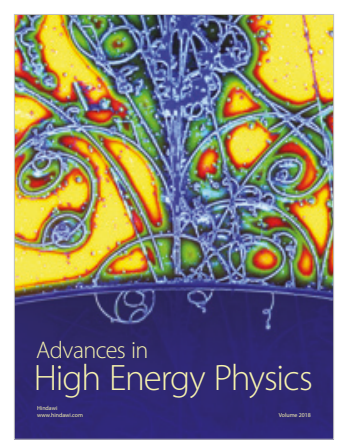

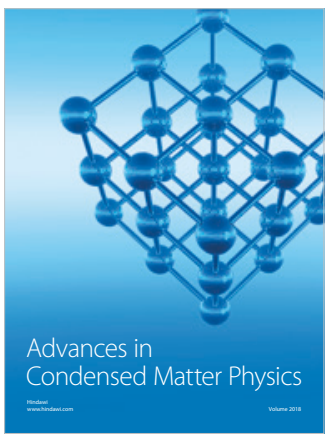

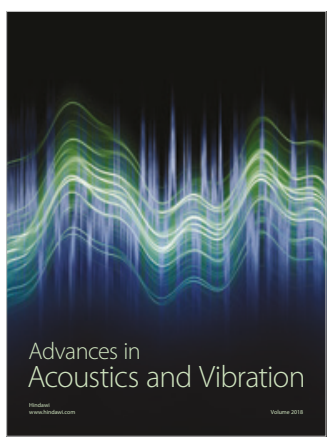

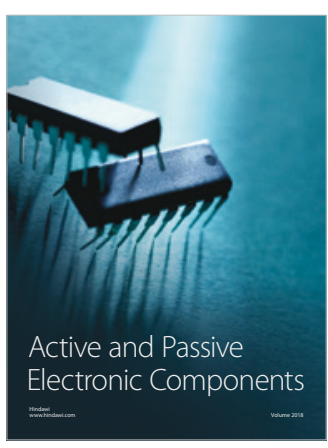
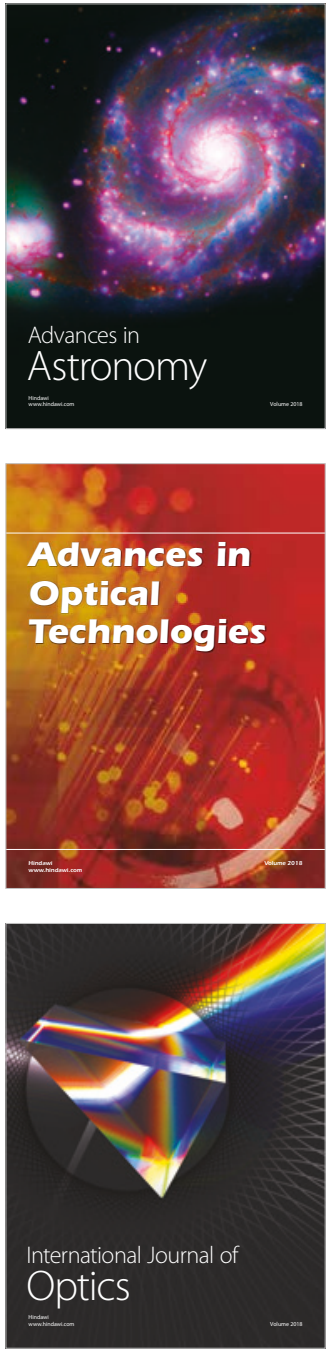